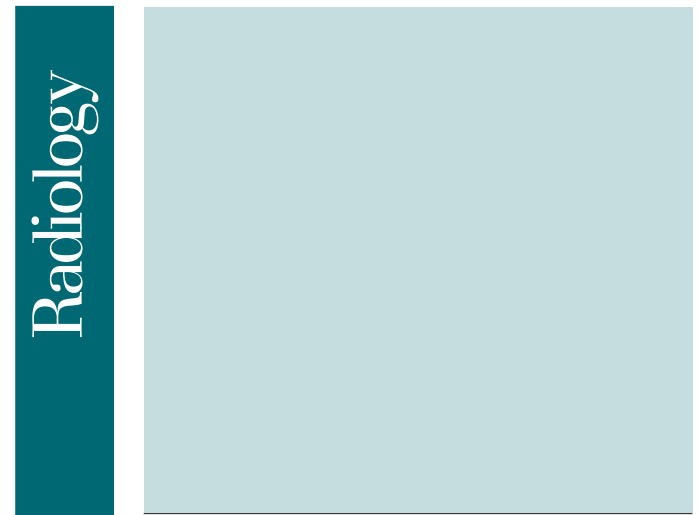

\title{
Thalamic Resting-State
} Functional Networks: Disruption in Patients with Mild Traumatic Brain<smiles>C1=CC=CC=CC=C1</smiles>

\section{Lin Tang, PhD}

Yulin Ge, MD

Daniel K. Sodickson, MD, PhD

Laura Miles, PhD

Yongxia Zhou, PhD

Joseph Reaume, BSRT

Robert I. Grossman, MD
${ }^{1}$ From the Center for Biomedical Imaging, Department of Radiology, New York University School of Medicine, 660 First Ave, 4th Floor, New York, NY 10016. Received January 3, 2011; revision requested February 8; revision received April 7; accepted April 14; final version accepted May 2. Address correspondence to Y.G. (e-mail: yulin.ge @nyumc.org)
Purpose:

Materials and Methods:

Results:

Conclusion:

These findings of abnormal thalamic RSNs lend further support to the presumed subtle thalamic injury in patients with MTBI. Resting-state functional MR imaging can be used as an additional imaging modality for detection of thalamocortical connectivity abnormalities and for better understanding of the complex persistent postconcussive syndrome.

${ }^{\circ}$ RSNA, 2011 resting-state functional magnetic resonance (MR) imaging and to investigate whether thalamic resting-state networks (RSNs) are disrupted in patients with mild traumatic brain injury (MTBI).

This HIPAA-compliant study was approved by the institutional review board, and written informed consent was obtained from 24 patients with MTBI and 17 healthy control subjects. The patients had varying degrees of symptoms, with a mean disease duration of 22 days. The restingstate functional MR imaging data were analyzed by using a standard seed-based whole-brain correlation method to characterize thalamic RSNs. Student $t$ tests were used to perform comparisons. The association between thalamic RSNs and performance on neuropsychologic and neurobehavioral measures was also investigated in patients with MTBI by using Spearman rank correlation. healthy subjects. This pattern was characterized as representing relatively symmetric and restrictive functional thalamocortical connectivity, suggesting an inhibitory property of the thalamic neurons during the resting state. This pattern was disrupted, with significantly increased thalamic RSNs $(P \leq .005)$ and decreased symmetry $(P=.03)$ in patients with MTBI compared with healthy control subjects. Increased functional thalamocortical redistributive connectivity was correlated with diminished neurocog nitive functions and clinical symptoms in patients with MTBI.

${ }^{\circ}$ RSNA, 2011 
F unctional magnetic resonance (MR) imaging studies of the neural functional network during the resting state have demonstrated highly correlated, slow fluctuations in blood oxygen level-dependent (BOLD) signal across discrete brain regions. Test-retest studies in healthy individuals have shown high reproducibility of these spatially coherent signal changes, which are more than simply random noise. In fact, they represent spontaneous and consistent fluctuations $(1,2)$. This intrinsically connected functional architecture, which is referred to as the resting-state network (RSN) (3), probably plays a role in maintaining baseline human cognition and metabolic equilibrium in the resting state. To date, resting-state functional MR imaging has been applied to several neuropsychiatric diseases, including attention deficit/hyperactivity disorder (4), depression (5), nicotine deactivation (6), schizophrenia (7), and Alzheimer disease (8), to help characterize the disturbances of the functional connectivity or networks (9).

Mild traumatic brain injury (MTBI) accounts for at least $75 \%$ of all traumatic brain injuries and is an important public health care problem (10). Patients with MTBI may initially experience a transient loss of consciousness ( $<30$ minutes) and/or a brief amnestic period $(<24$ hours) with disabling physical (headache, dizziness), cognitive (memory loss, attention deficit), and emotional and behavioral (depression, anxiety) sequelae that can persist for months or years (11). Despite such a disabling array of symptoms, which define postconcussive syndrome (PCS), in patients with MTBI

\section{Advances in Knowledge}

- Thalamic resting-state networks (RSNs) are disrupted in patients with mild traumatic brain injury (MTBI), suggesting that there may be reactive upregulation of thalamocortical connectivity associated with subtle thalamic injury.

- Injury to thalamic RSNs correlates with reduced performance in neurocognitive testing. there is typically an absence of obvious structural lesions, which has made it difficult to understand the pathophysiology of MTBI. Traditionally, the deficits associated with PCS have been assumed to be caused by diffuse axonal injury, particularly in moderate and severe traumatic brain injury; however, accumulating evidence is pointing toward thalamic injury as an important factor (12-16).

The thalamus, a centrally located relay station for transmitting information throughout the brain, participates in communication with many associative brain regions and involves global multifunctional pathways. It also contributes to the normal brain rhythms that are responsible for basic inhibitory control and coordination (17). Hence, the thalamocortical system is critical for integrating information across functional circuits. The thalamus is a complex deep gray matter mass that consists of many groups of nuclei and white matter bundles; it is vulnerable to damage during sudden acceleration or deceleration movements during head trauma. Injury involving thalamic neurons may alter the electroresponsiveness of the thalamocortical functional networks and cause a persistent state of abnormal excitation $(15,18)$, leading to a constellation of neuropsychiatric and behavioral symptoms or dysrhythmia. Despite the evidence in these studies that used electroresponsiveness as marker, imaging markers for thalamic injury are still missing, and little imaging evidence exists for the physiologic or structural origin of postconcussive disorders.

Brain functional RSNs represent a consistent set of highly correlated brain regions with respect to low-frequency BOLD activities during resting state (19). These resting activity correlations with classic motor or sensory cortical

\section{Implication for Patient Care}

- The observed disruption in thalamic functional RSNs in MTBI can be used for detecting subtle injury and to better understand the complex persistent postconcussive syndrome in these patients. gray matter regions and default mode networks have been well described in previous studies (3); however, the resting functional networks of deep gray matter structures, such as the thalamus, have been poorly studied with restingstate functional MR imaging. Moreover, there have been only a few reports of functional connectivity in patients with severe traumatic brain injuries $(20,21)$, and, to our knowledge, no resting-state functional MR imaging studies have been performed in MTBI. The analysis of intrinsic functional architectural changes by using resting-state functional MR imaging in MTBI may have great potential to improve the understanding of disease pathophysiology, aid accurate diagnosis, and monitor the effective treatment.

Our purpose was to explore the neural correlates of the thalamus by using resting-state functional MR imaging and to investigate whether thalamic RSNs are disrupted in patients with MTBI. We also investigated the association between thalamic connectivity changes and the performance on neuropsychologic and neurobehavioral tests in these patients. We hypothesized that there is subtle injury involving the thalamic neurons in patients with MTBI that may reconfigure

\section{Published online before print \\ 10.1148/radiol.11110014 Content code: NR \\ Radiology 2011; 260:831-840 \\ Abbreviations: \\ BOLD = blood oxygen level-dependent \\ $\mathrm{MTBI}=$ mild traumatic brain injury \\ PCS = postconcussive syndrome \\ $\mathrm{RSN}=$ resting-state network}

\section{Author contributions:}

Guarantors of integrity of entire study, L.T., Y.G.; study concepts/study design or data acquisition or data analysis/ interpretation, all authors; manuscript drafting or manuscript revision for important intellectual content, all authors; manuscript final version approval, all authors; literature research, L.T., Y.G., L.M., Y.Z., R.I.G.; clinical studies, L.T., Y.G., L.M., R.I.G.; statistical analysis, L.T., Y.G., Y.Z.; and manuscript editing, L.T., Y.G., D.K.S., L.M., Y.Z.

\section{Funding:}

This research was supported by the National Institutes of Health (grants R01NS039135, R01NS039135-08S1, R01NS029029, and R01HL069023).

Potential conflicts of interest are listed at the end of this article. 
the operations of intrinsic thalamocortical networks, as indicated at restingstate functional MR imaging.

\section{Materials and Methods}

\section{Subjects}

This study was approved by our institutional review board and was in compliance with the Health Insurance Portability and Accountability Act. After receiving an explanation of the study procedure, all of the participants provided written informed consent. From March 2008 to December 2009, a total of 27 patients and 20 age-matched healthy subjects were recruited for this prospective study in a university-affiliated level I trauma center and hospital (Bellevue Hospital and New York University Langone Medical Center, New York, NY). All patients with MTBI experienced a closed head injury with either posttraumatic amnesia of less than 24 hours or loss of consciousness of approximately 30 minutes or less. Their Glasgow Coma Scores were between 13 and 15. All patients, with a mean interval between MR imaging and trauma of 22 days (range, 3-53 days), reported various neurocognitive and neurobehavioral symptoms, including headache; dizziness; insomnia; fatigue; light sensitivity; irritability; and deficits in attention, concentration, memory, and executive function. Patients were recruited only after meeting the following inclusion criteria: no history of alcohol or drug abuse; no history of neuropsychologic diseases before injury; and no history of other neurologic diseases, including stroke, epilepsy, and somatic disorders. On the basis of these criteria, three patients were excluded because we later discovered that one had experienced previous head injuries, one had a drug habit, and one could not finish the neuropsychologic testing within 12 hours of MR imaging. In addition, three control subjects were excluded because of substantial motion during the MR imaging examination in two subjects and white matter lesions at MR imaging in one subject. Therefore, data in a total of 24 patients were used in this study (mean age, 37.3 years; range, 22-62 years). There were 17 male patients (mean age, 35.3 years; range, $22-56$ years) and seven female patients (mean age, 43.6 years; range, 25-62 years). A total of 17 healthy control subjects were ultimately included (mean age, 35.2 years; range, 23-49 years) for comparison.

\section{MR Imaging}

Functional MR imaging was performed by using a 3.0-T whole-body imaging unit (Siemens Magnetom Trio; Siemens Medical Solutions, Erlangen, Germany) and a gradient-echo echo-planar imaging sequence with the following parameters; repetition time sec/echo time msec, $2 / 30$; flip angle, $75^{\circ}$; field of view, $220 \times 220 \mathrm{~mm}^{2}$; and acquisition matrix, $128 \times 128$. Twenty 5 -mm-thick sections were acquired parallel to a line passing through the anterior-posterior commissure with a 1-mm gap and were positioned to cover nearly the entire cerebrum, resulting in a spatial resolution of $1.72 \times 1.72 \times 6 \mathrm{~mm}^{3}$ for functional MR imaging. After the functional MR imaging acquisition, to achieve better registration, a high-spatial-resolution wholebrain three-dimensional T1-weighted sagittal magnetization-prepared rapid acquisition gradient-echo sequence (repetition time msec/echo time msec, 2300/2.98; inversion time, $900 \mathrm{msec}$; flip angle, $9^{\circ}$; spatial resolution, $1 \times 1 \times$ $1 \mathrm{~mm}^{3}$ ), as well as a two-dimensional T1-weighted acquisition at the identical section positions, were performed. In addition, a high-spatial-resolution susceptibility-weighted imaging sequence and a T2-weighted fast spin-echo sequence were also performed to supplement the studies and to help detect if any hemorrhagic or other lesions were present. The imaging parameters of the susceptibility-weighted imaging sequence were as follows: repetition time $\mathrm{msec} /$ echo time msec, 45/20; flip angle, $15^{\circ}$; matrix, $448 \times 320$; field of view, $220 \times$ $220 \mathrm{~mm}^{2}$; and section thickness, $2 \mathrm{~mm}$. The imaging parameters of the T2weighted fast spin-echo sequence were as follows: repetition time msec/echo time msec, 5000/88; flip angle, $120^{\circ}$; matrix, $448 \times 364$; field of view, $220 \times$ $220 \mathrm{~mm}^{2}$; and section thickness, $3 \mathrm{~mm}$.
All patients and healthy subjects were instructed to close their eyes but stay awake during the resting-state functional MR imaging examination, which was the same for patients and control subjects. So that we could test repeatability, two resting-state MR imaging sessions, separated by 15 minutes of anatomic imaging, were performed in only healthy subjects. Each functional MR imaging session obtained 153 volumes and lasted 5 minutes and 6 seconds. Repeat resting-state functional MR imaging was also performed in healthy volunteers on different days separated by a minimum of 4 weeks so that we could check intrasubject reliability over time.

\section{Neuropsychologic Assessment}

Neuropsychologic tests were administered within 12 hours of functional MR imaging in patients with MTBI by a psychologist (L.M.) in the Department of Psychology at the Rusk Institute of Rehabilitation Medicine at the New York University School of Medicine. The psychologist had more than 7 years of experience in neuropsychologic test design and measurement and was blinded to the functional MR imaging results and study subject allocation (patients vs healthy control subjects). Neuropsychologic measures assessed areas of cognitive functioning found to be impaired in patients with MTBI. Test results were reported in terms of standardized $z$ scores (mean score, $0 \pm 1$ [standard deviation]). The Digit Span subtest of the Wechsler Adult Intelligence Scale III (22) was used to measure verbal attention and concentration, as well as working memory. Trail-making test A (23) was used to assess speed and visual attention, and trail-making test B (23) was used to assess mental flexibility, specifically the ability to shift rapidly between cognitive sets, a measure of executive functioning. The Paced Auditory Serial Addition Test (24) was used to assess sustained attention and concentration, as well as working memory. The Symbol Digit Modalities Test (25) measured information processing speed. Verbal learning and immediate and delayed verbal memory were assessed by using the California Verbal Learning Test II (26). 
Visuoperceptual ability and immediate and delayed visual memory were assessed with the Rey Complex Figure Test (27).

Postconcussive symptoms, depression, and fatigue were assessed by using self-report questionnaires, for which higher scores indicate greater symptoms. The Beck Depression Inventory (28) was used to assess depressive symptoms, fatigue was measured by using the $\mathrm{Fa}^{-}$ tigue Severity Scale (29), and subjective symptoms associated with MTBI were assessed with the Postconcussive Symptoms Scale (PSS) (30). The PSS assesses the severity of 19 symptoms (eg, dizziness, balance problems, headache, sensitivity to light) associated with PCS and/or mild cognitive impairment. Items are rated on a Likert scale indicating the severity of the symptom, from 0 (none) to 6 (severe). Scores range from 0 to 114 and are calculated by adding the endorsed item ratings, with higher scores indicating more symptoms associated with PCS. Past research has validated the use of such inventories in assessing individuals after minor head injuries (31). Additionally, many studies have identified the relationship between scores in such inventories and cognitive impairment after minor head injury (32-35).

\section{Image Processing and Data Analysis}

All functional MR imaging data were analyzed by using software (SPM2, Statistical Parametric Mapping, http://www fil.ion.ucl.ac.uk/; and MRIcro, http: //www.mricro.com/) and additional inhouse programs running in MATLAB (Mathworks, Natick, Mass). Preprocessing of functional MR imaging data was performed by using SPM2 and included motion correction (any data set with maximum head motion $>2 \mathrm{~mm}$ or $0.5^{\circ}$ was discarded), realignment, and coregistration with the T1-weighted images collected by using identical section prescriptions as well as with the magnetization-prepared rapid acquisition gradient-echo whole-brain structural images. Then, all brain image volumes were spatially normalized (36) into a standard space defined by the Montreal Neurologic Institute template in standard Talaraich coordinates. Hence, after normalization, the real brain can be seen as an alias volume with each voxel sized at $2 \times 2 \times 2 \mathrm{~mm}^{3}$ and standard coordinates based on the Talaraich Daemon, with corresponding brain functional areas as defined. Subsequently, the data sets were spatially smoothed with an isotropic Gaussian kernel with a full width at half maximum of $6 \mathrm{~mm}$ to compensate for intersubject gyral variability and to attenuate high-frequency noise, thus increasing the signal-to-noise ratio. SPM2 estimated a resulting smoothness of $8.2 \times 8.4 \times 8.7 \mathrm{~mm}^{3}$. The first three volumes of each functional time series were discarded.

In individual resting-state functional MR imaging data analysis, we used bilateral thalami as seed regions. These regions were selected with the help of the WFU PickAtlas tool (http://fmri .wfubmc.edu/downloads/WFU_Pick Atlas_User_Manual.pdf). The seed regions were used to define the reference time course; then the cross-correlation coefficient ( $R$ score) between each reference time course and the signal time series in each voxel within the acquired whole-brain image set was computed. Functional connectivity maps for each study subject were then generated by displaying all those voxels whose signal time series were significantly correlated with the seed regions $(P<.001$, corresponding to $R>0.25)$. The total number of voxels from connectivity maps for each study subject's left, right, and bilateral thalamic RSNs were then calculated. We used the percentage of the overlap of the two thalamic RSNs to study the conjugate symmetry property between the left and right thalamic RSNsthat is, the percentage of the total voxel number of bilateral thalamic RSNs that correlated with not only the left but also the right thalamus. Hence, a higher overlapping percentage represents higher symmetry between the left and right thalamic RSNs. Finally, we performed a Z-transform of the voxel numbers of thalamic RSNs in patients with MTBI by using the mean and standard deviation in the healthy control subjects to standardize the statistical analysis. The calculated Z scores in patients with MTBI were correlated with their neurocognitive and neurobehavioral measures to evaluate our method of using resting-state functional networks of thalamocortical connectivity to detect the brain functional abnormalities behind the clinical symptoms.

In addition to the seed-based analysis, we performed multiple subject group independent component analysis by using multisession temporal concatenation with identification of group-wise thalamocortical default-mode connectivity by using FSL (FMRIB Software Library, http://www.fmrib.ox.ac.uk/fsl/) MELODIC (Multivariate Exploratory Linear Optimized Decomposition into Independent Components) on the basis of the component's power spectrum, low-frequency fluctuation, and overall spatial pattern. The thalamus, based on the FSL Juelich template, was then correlated with each decomposed MELODIC component. Each correlation between the associated time course and average time course of the thalamus was computed, and a component was identified for the thalamus on the basis of the highest correlation among all the components.

\section{Statistical Analysis}

In group analysis, the Fisher z' transformation was introduced to compute Fisher $z$ ' maps for each study subject because the correlation coefficient $R$ is not normally distributed. One-sample $t$ tests within the groups were then performed to generate group RSN maps showing all areas with cluster sizes larger than 100 voxels that were significantly correlated with the seed regions of interest $(P<.001)$ for both healthy control subjects and patients (37). Twosample $t$ tests were then performed to quantitatively demonstrate the difference between the RSNs of patients with MTBI and those of healthy control subjects, and the results were displayed as comparison maps. The statistical significance levels were determined by $P$ values, and the comparison significance level was set at uncorrected $P<.01$ and cluster size $k>100$ voxels.

To evaluate our method of using resting-state functional networks of thalamocortical connectivity to detect the brain functional abnormalities behind 
clinical symptoms, we evaluated the relationship between the quantitative thalamic RSNs in the patients with MTBI and their neurocognitive and neurobehavioral measures by using Spearman and Pearson correlations to calculate the correlation coefficient in the patient group. In addition, we performed Bonferroni adjustment for multiple comparisons for these correlations $(t>3.12, P<.005)$.

\section{Results}

No lesions were seen in the thalamic regions on either conventional T2-weighted or susceptibility-weighted structural images in any subject. In healthy control subjects at rest, a normal pattern of thalamic RSNs was observed that primarily included areas of the bilateral thalamus, the superior frontal gyrus, the middle frontal gyrus, the basal ganglia nuclei, the insula, and the cingulate gyrus (Fig 1). This pattern was consistent when the seed region was selected in either the right or left side of the thalamus, showing no significant difference in clusters of voxels between the ipsilateral and the contralateral hemisphere (two-tailed $P=$ .98 , paired $t$ test). In addition, when the test-retest reliability was evaluated in healthy subjects, the thalamic RSNs showed a reliable pattern of functional connectivity in the left and right hemispheres and bilaterally (two-tailed $P>$ .8) across different sessions, including resting-state functional MR imaging data sets acquired on the same day separated by 15 minutes and data sets acquired on different days.

Compared with control subjects, patients with MTBI demonstrated more widely distributed functional connectivity between thalamic and cortical regions during the resting state (Table 1), despite the fact that no lesions were found in thalamic regions at conventional imaging. Specifically, the thalamic RSNs $(P<.001$, corresponding to $R>$ 0.25 ) in patients with MTBI extended to the bilateral middle temporal gyrus, the middle frontal gyrus, the precuneus, the inferior parietal gyrus, and the postcentral gyrus (Fig 2, $A$ and $B$ ), compared with the thalamic RSNs in the healthy control subjects (Fig 2, $B$ and $C$ ). The
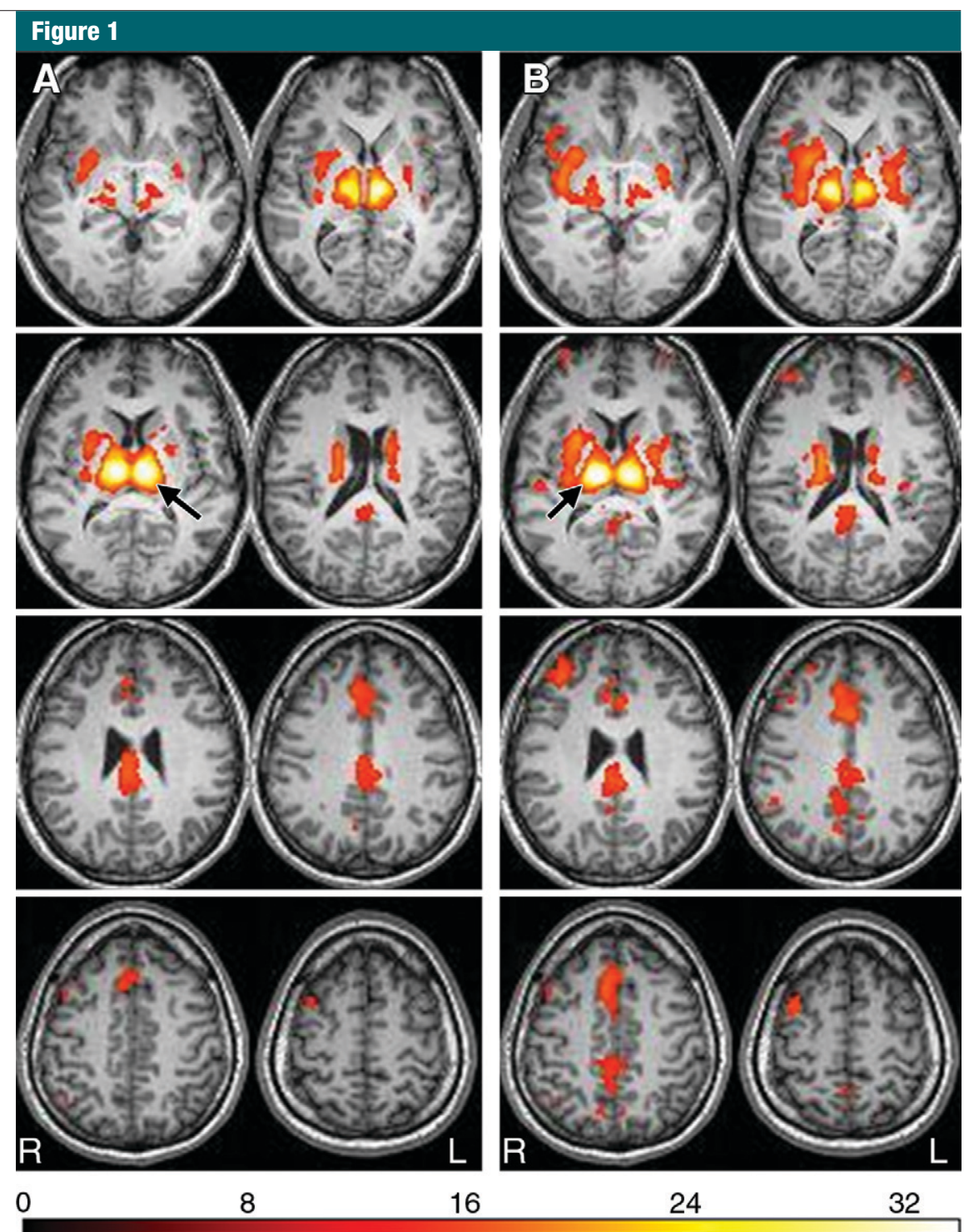

0

8

16

Figure 1: Multiple-section axial views of MR imaging data show the normal pattern of thalamic RSNs in the healthy control group when the seed (arrows) was placed in, $A$, the left and, $B$, the right thalamus. The regions highlighted in the thalamic RSNs primarily involved the thalami bilaterally (with the highest correlation coefficient), the inferior frontal gyrus, the superior temporal gyrus, the basal ganglia nuclei, the insula, the cingulate gyrus, and the supramarginal gyrus. $L=$ left, $R=$ right.

increased functional connectivities in the patient group or the difference $(P<$ $.01)$ between the two groups involved regions of the cingulate gyrus, temporal gyrus, and frontal gyrus (Fig 2, E and $F$ ). For individual thalamic functional RSNs, when the threshold of the correlation coefficient $R$ was increased from 0.25 , which corresponds to a criterion of $P<.001$, to a much stricter criterion of 0.6 , we found that the RSNs in all control subjects were restricted to only the thalami bilaterally, but patients still showed a widespread pattern, with similar cortical regions involved. This indicates that, in patients with MTBI, both the extent and the correlation strength of the thalamocortical functional connectivity are increased. At MELODIC independent component analysis, patients 
Table 1

\section{Brain Areas Involved in Left Thalamic RSNs in Healthy Control Subjects and Patients with MTBI}

\begin{tabular}{|c|c|c|c|c|c|c|c|}
\hline \multirow[b]{2}{*}{ Brain Area } & \multirow[b]{2}{*}{ Brodmann Area } & \multicolumn{3}{|c|}{ Healthy Control Subjects } & \multicolumn{3}{|c|}{ Patients with MTBI } \\
\hline & & $x, y, z(\mathrm{~mm})$ & kValue & $t$ Value & $x, y, z(\mathrm{~mm})$ & $k$ Value & $t$ Value \\
\hline Left thalamus & $\ldots$ & $6,-16,8$ & 4780 & 33.86 & $12,-16,8$ & $4198 *$ & 33.41 \\
\hline Right thalamus & $\ldots$ & $-10,-14,4$ & 4053 & 29.71 & $-10,-14,4$ & $4198^{\star}$ & 27.27 \\
\hline $\begin{array}{l}\text { Left basal ganglia, insula, superior } \\
\text { temporal gyrus }\end{array}$ & $13 / 22$ & $28,-12,10$ & 2057 & 17.64 & $36,-20,4$ & $4198^{\star}$ & 7.64 \\
\hline $\begin{array}{l}\text { Right basal ganglia, insula, superior } \\
\text { temporal gyrus }\end{array}$ & $13 / 22$ & $-28,-18,8$ & 3027 & 18.23 & $-38,-22,12$ & $4198^{\star}$ & 10.64 \\
\hline Anterior cingulate gyrus & 8 & $6,14,26$ & 259 & 6.92 & $-2,32,58$ & 1007 & 5.17 \\
\hline Precuneus, posterior cingulate gyrus & $7 / 31$ & $-6,-34,24$ & 430 & 6.64 & $6,-78,38$ & 959 & 6.38 \\
\hline Cingulate gyrus & 24 & & & & $0,-22,38$ & 1677 & 5.65 \\
\hline Middle temporal gyrus & 21 & & & & $-54,-48,-2$ & 106 & 4.47 \\
\hline Superior frontal gyrus, middle frontal gyrus & $9 / 10$ & & & & $42,44,38$ & 105 & 8.39 \\
\hline Inferior parietal gyrus & 40 & & & & $-58,-40,30$ & 120 & 5.35 \\
\hline Postcentral gyrus & 3 & & & & $24,-35,54$ & 146 & 4.42 \\
\hline
\end{tabular}

Note $-k$ Value $=$ cluster size (number of voxels); $t$ value $=$ that of local maximum; $x, y, z=$ Montreal Neurological Institute coordinates. The $t$ values; $x, y, z$ coordinates; and Brodmann areas are listed for the local maxima reaching the highest $t$ values. Absent values for areas in healthy control subjects indicate no thalamic functional connectivity in these regions.

* When clusters grow too large or come too close to each other to be separated, a specific or minimal number (ie, $k=4198$ ) is provided by the software, indicating that the cluster is larger than that number.

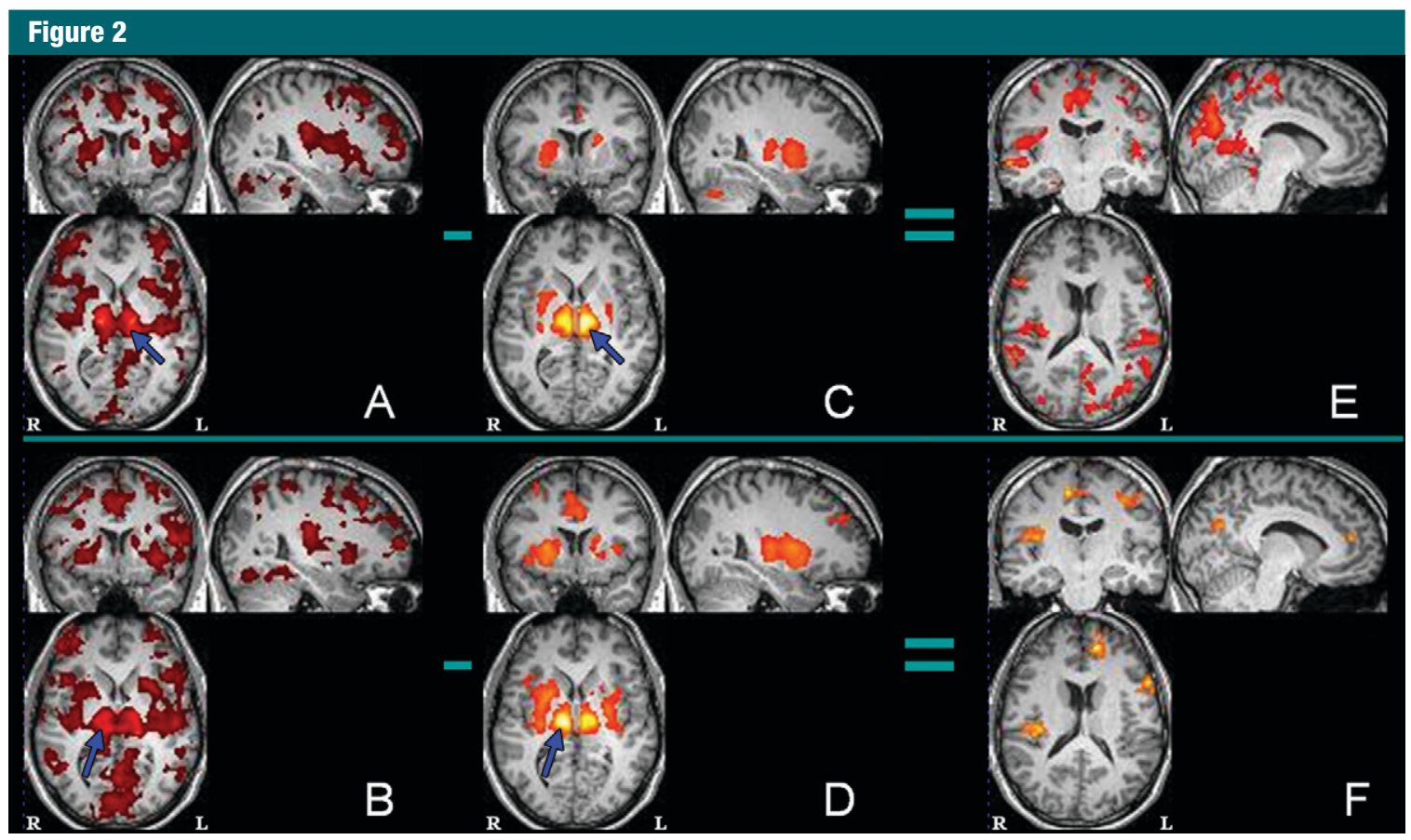

Figure 2: Images show group thalamic RSNs in 3D views obtained by using the one-sample $t$ test $(P<.001, k>100)$ in, $A, B$, patients with MTBI and, $C, D$, control subjects. $E$, $F$, The difference maps between the two groups (patient - control) were compared by using the two-sample $t$ test $(P<.01, k>100)$. Arrows = seed placement locations; the patterns of the left $(L)$ and right $(R)$ thalamic functional networks during the resting state are shown in the first and second rows, respectively. Compared with healthy control subjects, patients with MTBI showed more widely distributed thalamic functional connectivity during the resting state, with increases in regions involving the cingulate, temporal, and frontal gyri.

with MTBI also showed significantly increased thalamocortical connectivities in the posterior cingulate and frontal regions compared with healthy control subjects $(P<.05)$, which is consistent with the results of seed-based analysis.
Quantitative analysis of the total number of voxels involved in thalamic RSNs showed significantly higher voxel 
numbers in patients with MTBI than in healthy control subjects when the seed was placed in either side of the thalamus or in both thalami $(P \leq .005)$ (Table 2 , Fig 3). The thalamic RSNs during the resting state in patients with MTBI were made up principally of positive correlations, indicating that these regions tend to be correlated in their spontaneous BOLD signal with similar functionality. In the group analysis, thalamic functional RSNs were generally inhomogeneous and were more widely spread in patients than in control subjects (Fig 3). In contrast to a fair degree of symmetry between left and right thalamic RSNs patterns in healthy control subjects (Fig 1), in patients with MTBI, there was significantly decreased symmetry of the left and right thalamic RSNs $(P=.03)$, defined as the percentage of overlaid voxels among the total number of voxels from both sides (Fig 4).

In patients with MTBI, a significant negative correlation $(P<.05)$ between results on the Rey Complex Figure Test, a neurocognitive test associated with working memory, and the total involved voxel numbers of both left and right thalamic RSNs was found by using the Spearman rank correlation coefficient ( $\rho=-0.55$, one-tailed $P=.003$ ) (Fig 5a), indicating that patients with worse visuospatial ability and memory showed increased voxel numbers of thalamic RSNs. There was a negative correlation between the symmetry of thalamic RSNs (percentage overlap) and the degree of symptoms in patients with MTBI. Specifically, patients who reported higher levels of depression $(\rho=-0.52, P=.005)$ or postconcussive symptoms $(\rho=-0.45$, $P=.014)$ (Fig $5 b)$ and older patients $(\rho=$ $-0.57, P=.002$ ) showed a lower degree of symmetry of the thalamic RSNs. After Bonferroni adjustment, older patients and patients with higher levels of depression still showed significantly lower symmetry of the thalamic RSNs. These results suggest a relationship between the degrees of compensation for restoration (ie, increased thalamic RSNs) associated with subtle brain damage in thalamocortical connectivity and neurocognitive ability, particularly that associated with working memory, in our

\begin{tabular}{|c|c|c|c|}
\hline \multicolumn{4}{|c|}{$\begin{array}{l}\text { Voxel Numbers of Thalamic RSNs in Patients with MTBI }(n=24) \text { and Healthy Control } \\
\text { Subjects }(n=17)\end{array}$} \\
\hline Seed Location for Thalamic RSNs & Control Subjects & Patients with MTBI & $P$ Value ${ }^{*}$ \\
\hline Left thalamus & $71234 \pm 27399$ & $102480 \pm 33489$ & .001 \\
\hline Right thalamus & $78126 \pm 29063$ & $103060 \pm 28865$ & .005 \\
\hline Both & $89180 \pm 32505$ & $130776 \pm 34905$ & .0002 \\
\hline
\end{tabular}

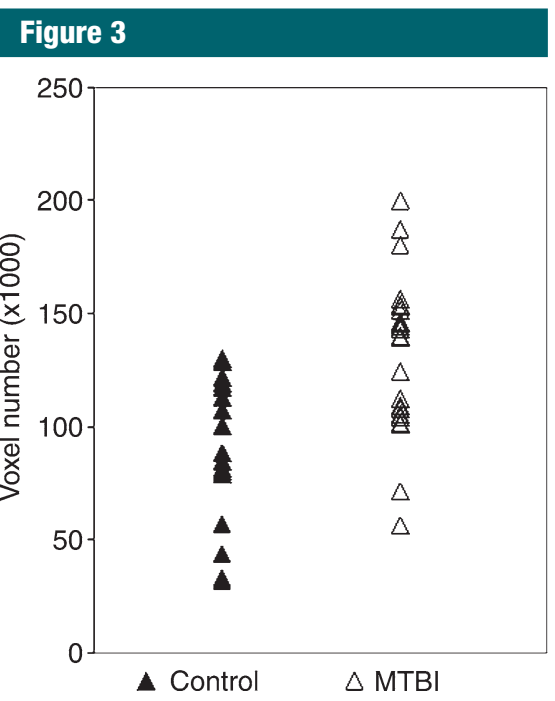

Figure 3: Graph of comparison of total voxel numbers shows significant difference $(P=.0002)$ in the bilateral thalamic RSNs between healthy volunteers and patients with MTBI.

patient group. No correlations were found between abnormal thalamic RSNs and other neurocognitive scores in these patients.

\section{Discussion}

There is growing interest in examining patients with MTBI to elucidate the pathophysiologic mechanisms underlying a variety of posttraumatic symptoms. Our current study results indicate that patients with MTBI suffere from disruption in their thalamic RSNs, manifest as reactive upregulation of thalamocortical functional connectivity; furthermore, these abnormal thalamic RSNs are associated with decreased neurocognitive performance. Because these findings have not previously been well established in

\section{Figure 4}

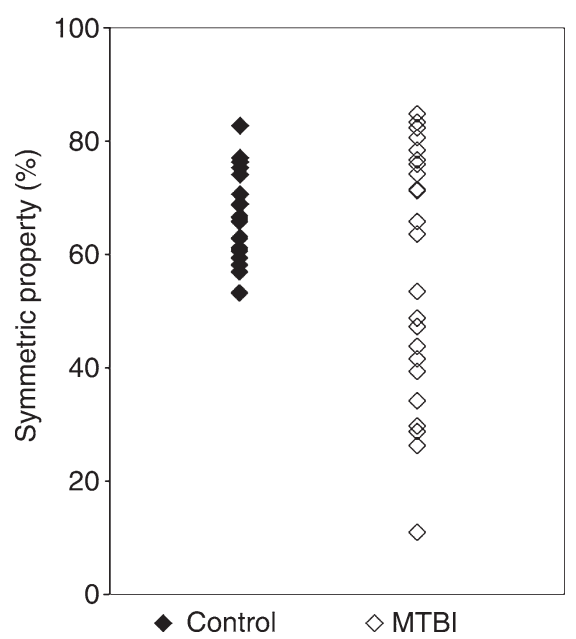

Figure 4: Graph of comparison of symmetry between patients with MTBI and healthy control subjects shows significantly lower symmetry in patients $(P=.03)$. The symmetric property is defined as the degree of overlay between the left thalamic RSN and the right thalamic RSN and is expressed as a percentage.

the literature, the observations of this study suggest that resting-state functiona MR imaging may have the potential to provide objective measures in patients with MTBI and posttraumatic symptoms that are otherwise difficult to explain with conventional MR imaging findings.

Using experimental and established neuropsychologic tests, previous investigators $(38,39)$ have demonstrated that the thalamus plays a crucial role in memory, executive functioning, and attention. Owing to the complicated nature of traumatic brain injury and the uneven densities of the internal nuclei, the thalamus, a large, central gray matter mass, is particularly vulnerable during head injury. It has been confirmed by several 


\section{Figure 5}
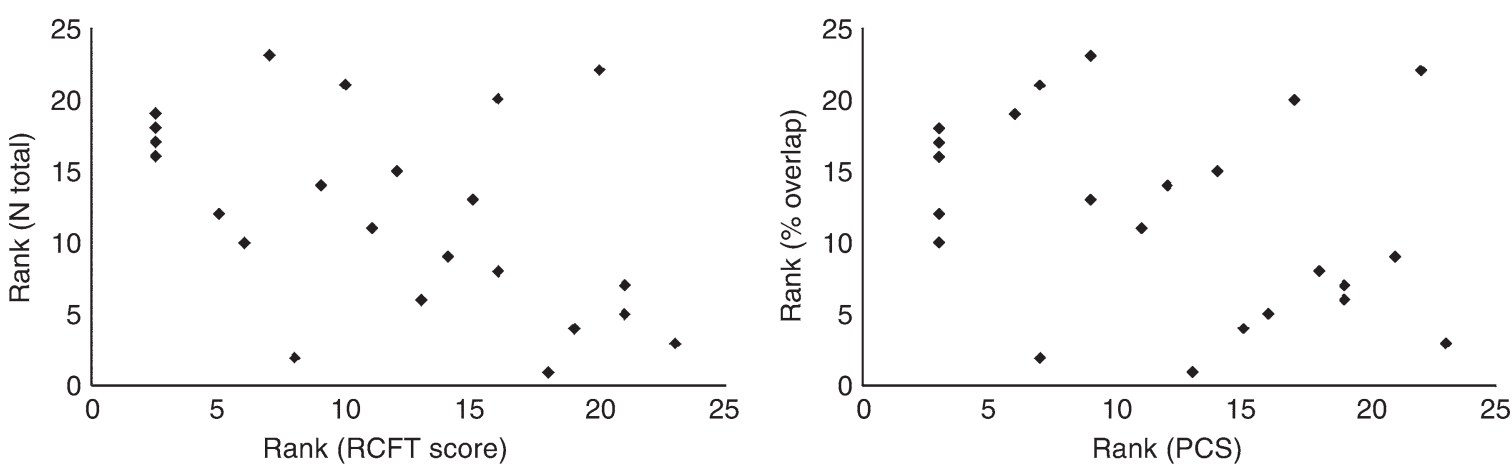

b.

Figure 5: Graph shows Spearman rank correlations between patients' neurocognitive tests or symptoms and quantitative thalamic RSNs for

(a) correlation between total voxel number of both thalamic RSNs and Rey Complex Figure Test (RCFT) scores $(\rho=-0.55, P=.003)$ and

(b) correlation between symmetry of thalamic RSNs and level of PCS $(\rho=-0.45, P=.014)$.

histopathologic studies $(12,13,16)$ that there is thalamic injury and neuronal loss in the thalamic nuclei during traumatic brain injury. However, these previous studies examined only patients with moderate to severe disabilities, unlike the mild disabilities in our current study. Conventional imaging modalities typically fail to depict abnormalities in MTBI. Using advanced quantitative techniques such as diffusion-tensor imaging, Little et al (40) showed reductions in fractional anisotropy in several white matter regions when fiber tracking seeds were placed in the thalamic nuclei in a mixed group of patients with mild and moderate to severe injury. Other studies involving diffusion-tensor imaging and proton MR spectroscopy $(41,42)$ revealed only minimal detectable differences in the thalamus between the MTBI and control groups. Ge et al (43) recently reported decreased cerebral flow in the thalamus in MTBI, suggesting blood flow compromise in these patients. Owing to the thalamus's critical role in brain function and an increased awareness of the cognitive sequelae in patients with MTBI, it is vital to develop a sensitive and objective imaging tool that allows researchers to better detect thalamic injury, which is considered to be subtle and largely underestimated in MTBI. This may be also useful for distinguishing between symptoms from an injury and factitious or malingering symptoms.
Our results demonstrated a normal pattern of thalamic functional RSNs in healthy volunteers that primarily involves the thalami bilaterally with the highest homogeneity and strength, as well as other cortical and subcortical regions, including the superior frontal gyrus, the middle frontal gyrus, the basal ganglia nuclei, the insula, and the cingulate gyrus, which showed a relatively higher symmetric pattern. The test-retest analysis showed a high repeatability and results consistent with the findings of a previous study (44). Our results for thalamic RSNs were based on the resting-state functional MR imaging voxels meeting a criterion of correlation significance of $P<.001$, in contrast to the $P<.05$ used in a previous study (45), in which more cortical regions were identified. A relatively higher or stricter significance level was selected for the current study to obtain higher specificity with increased correlation strength for better comparison of the thalamic RSNs between patients and control subjects. When an even stricter criterion was applied in healthy control subjects, only bilateral thalami were shown, indicating that the strength of functional connectivity within the bilateral thalamic nuclei is much higher than within the thalamocortical networks.

The increased thalamocortical connectivity or thalamic RSNs in patients as compared with control subjects remained significant even after the corre- lation threshold was raised from $R=0.25$ to $R=0.6$. These experimental findings may have several implications about thalamocortical functional networks in patients with MTBI. First, the low frequency BOLD fluctuations of resting-state functional MR imaging in the cortex are typically in the range of $0.01-0.10 \mathrm{~Hz}$ and were shown to reflect functional connectivity. The increment of the thalamocortical correlation of the BOLD fluctuations in patients with MTBI can arise from the aberrant frequency distribution of low-frequency fluctuations in the thalamus. Second, the rich GABAergic neurons of the thalamic reticular nucleus act as inhibitory intermediaries, not only acting within thalamic nuclei but also between thalamic nuclei and thalamocortical neurons $(46,47)$, with the consequence that the thalamocortical functional networks are only loosely connected in the normal resting condition. In MTBI, a reactive upregulation can occur when GABAergic neurons are damaged, leading to an increased synchronization with other brain regions. In previous study of choreic Huntington disease (46), this disinhibited pathway in the caudate nucleus and thalamus was also considered to be responsible for the overexcited thalamocortical output in the disease. Finally, the greater scattering of thalamic functional connectivity in patients with MTBI, who have a relatively short disease duration $(<60$ days) among patients with traumatic brain 
injury, indicates subtle acute or subacute thalamic injury. Nerve tissue injuries can be compensated by regenerating injured axons or by collateral branching of undamaged axons and by remodeling of nervous system circuitry (48). Animal studies $(49,50)$ have also shown considerable thalamic functional reorganization after peripheral nerve injury. This has also been shown to occur by increasing hemodynamic supply to compensate for the inefficient thalamic-cortical processing in cocaine abusers (51).

The patients in our study displayed a constellation of symptoms marked by headache, fatigue, cognitive deficits, and behavioral problems, which might be associated with thalamocortical dysrhythmia. The sensitivity of thalamocortical connectivity to cognitive ability was found to be higher in neurocognitive measures related to working memory in our patient group. In addition, the decreased degree of asymmetry between the left and right thalamic RSNs in patients with MTBI was also found to be correlated with clinical symptoms such as PCS scores and depression, which can also provide preliminary impairment evidence in the neurocircuit. Our findings suggest that the disrupted inhibitory properties of thalamic nuclei secondary to subtle injury resulted in upregulated thalamocortical connectivity in patients with MTBI. Although the possibility of thalamic network compensatory mechanisms has been previously suggested and supported by some functional imaging studies and by results of event-related potential investigations in patients with head injuries (52-54), the mechanism for thalamic functional compensation is still largely unclear in brain trauma. Investigating these abnormal thalamic RSNs and clinical posttraumatic symptoms in a longitudinal design will better illustrate the underlying mechanisms.

A few potential limitations of the current study should be examined. First, this study mainly focused on the thalamic functional network in patients with MTBI, and it should be pointed out that other RSNs may also be abnormal. A recent study of patients in the vegetative state (55) found marked dysfunctional default mode network abnormalities. These additional networks are being investigated in separate ongoing studies. Second, the seed used in this study was placed in the central thalamus and covered the thalamus partially; however, seeds corresponding to the thalamic subnuclei may improve the specificity of the analysis. This warrants further investigation. In the current study, we performed MELODIC independent component analysis and demonstrated a similar pattern of thalamic RSNs changes in patients with MTBI. Third, our study results do not disprove the possibility of structural changes in the thalamus, which can be measured by quantitative methods such as diffusion-tensor imaging. Future studies exploring the relationship between structural and functional network deficits and between these abnormalities and clinical posttraumatic symptoms in a longitudinal design will be valuable.

In summary, resting-state functional MR imaging can be used as an additional imaging modality for detection of subtle thalamic injury and for elucidating the pathophysiology behind persistent PCS in patients with MTBI.

Acknowledgment: Special thanks to KellyAnne McGorty, RT, for assistance in sequence preparation and imaging.

Disclosures of Potential Conflicts of Interest: L.T. No potential conflicts of interest to disclose. Y.G. No potential conflicts of interest to disclose. D.K.S. No potential conflicts of interest to disclose. L.M. No potential conflicts of interest to disclose. Y.Z. No potential conflicts of interest to disclose. J.R. No potential conflicts of interest to disclose. R.I.G. No potential conflicts of interest to disclose.

\section{References}

1. Damoiseaux JS, Rombouts SA, Barkhof F, et al. Consistent resting-state networks across healthy subjects. Proc Natl Acad Sci U S A 2006;103(37):13848-13853.

2. Meindl T, Teipel S, Elmouden R, et al. Testretest reproducibility of the default-mode network in healthy individuals. Hum Brain Mapp 2010;31(2):237-246.

3. Fox MD, Raichle ME. Spontaneous fluctuations in brain activity observed with functional magnetic resonance imaging. Nat Rev Neurosci 2007;8(9):700-711.
4. Zhu CZ, Zang YF, Cao QJ, et al. Fisher discriminative analysis of resting-state brain function for attention-deficit/hyperactivity disorder. Neuroimage 2008;40(1):110-120.

5. Greicius MD, Flores BH, Menon V, et al. Resting-state functional connectivity in major depression: abnormally increased contributions from subgenual cingulate cortex and thalamus. Biol Psychiatry 2007;62(5):429-437.

6. Hahn B, Ross TJ, Yang Y, Kim I, Huestis MA, Stein EA. Nicotine enhances visuospatial attention by deactivating areas of the resting brain default network. J Neurosci 2007; 27(13):3477-3489.

7. Zhou Y, Liang M, Jiang T, et al. Functional dysconnectivity of the dorsolateral prefrontal cortex in first-episode schizophrenia using resting-state fMRI. Neurosci Lett 2007; 417(3):297-302.

8. Sorg C, Riedl V, Mühlau M, et al. Selective changes of resting-state networks in individuals at risk for Alzheimer's disease. Proc Natl Acad Sci U S A 2007;104(47):18760-18765.

9. Fox MD, Greicius M. Clinical applications of resting state functional connectivity. Front Syst Neurosci 2010;4:19.

10. National Center for Injury Prevention and Control. Report to Congress on mild traumatic brain injury in the United States: steps to prevent a serious public health problem. Atlanta, Ga: Centers for Disease Control and Prevention, 2003

11. Bigler ED. Neuropsychology and clinical neuroscience of persistent post-concussive syndrome. J Int Neuropsychol Soc 2008; 14(1):1-22

12. Ross DT, Graham DI, Adams JH. Selective loss of neurons from the thalamic reticular nucleus following severe human head injury. J Neurotrauma 1993;10(2):151-165.

13. Maxwell WL, Pennington K, MacKinnon MA, et al. Differential responses in three thalamic nuclei in moderately disabled, severely disabled and vegetative patients after blunt head injury. Brain 2004;127(Pt 11):2470-2478.

14. Natale JE, Cheng Y, Martin LJ. Thalamic neuron apoptosis emerges rapidly after cortical damage in immature mice. Neuroscience 2002;112(3):665-676.

15. Schiff ND, Giacino JT, Kalmar K, et al. Behavioural improvements with thalamic stim ulation after severe traumatic brain injury. Nature 2007;448(7153):600-603.

16. Anderson CV, WoodDM, BiglerED, Blatter DD Lesion volume, injury severity, and thalamic integrity following head injury. J Neurotrauma 1996;13(2):59-65.

17. Llinás RR, Ribary U, Jeanmonod D, Kronberg E, Mitra PP. Thalamocortical dysrhythmia: 
a neurological and neuropsychiatric syndrome characterized by magnetoencephalography. Proc Natl Acad Sci U S A 1999;96(26): 15222-15227.

18. Welsh RC, Chen AC, Taylor SF. Low-frequency BOLD fluctuations demonstrate altered thalamocortical connectivity in schizophrenia. Schizophr Bull 2010;36(4):713-722.

19. Biswal B, Yetkin FZ, Haughton VM, Hyde JS. Functional connectivity in the motor cortex of resting human brain using echo-planar MRI. Magn Reson Med 1995;34(4):537-541.

20. Boly M, Phillips C, Balteau E, et al. Consciousness and cerebral baseline activity fluctuations. Hum Brain Mapp 2008;29(7):868-874.

21. Nakamura T, Hillary FG, Biswal BB. Resting network plasticity following brain injury. PLoS ONE 2009;4(12):e8220.

22. Wechsler D. Wechsler Adult Intelligence Scale III. New York, NY: The Psychological Corporation, 1997.

23. Reitan RM. Trail-making test: manual for administration and scoring. Tucson, Ariz: Reitan Neuropsychology Laboratory, 1992.

24. Gronwall D, Wrightson P. Recovery after minor head injury [letter]. Lancet 1974; 2(7894):1452.

25. Smith A. Symbol Digit Modalities: test manual. Los Angeles, Calif: Western Psychological Services, 1973.

26. Delis DC, Kramer JH, Kaplan E, Ober BA. California Verbal Learning Test: adult version. San Antonio, Tex: The Psychological Corporation, 2000 .

27. Meyers JE, Meyers KR. Rey Complex Figure Test and Recognition Trial: professional manual. Odessa, FL: Psychological Assessment Resources, 1995.

28. Beck AT, Ward CH, Mendelson M, Mock J, Erbaugh J. An inventory for measuring depression. Arch Gen Psychiatry 1961;4:561-571.

29. Krupp LB, LaRocca NG, Muir-Nash J, Steinberg AD. The fatigue severity scale: application to patients with multiple sclerosis and systemic lupus erythematosus. Arch Neurol 1989;46(10):1121-1123.

30. Aubry M, Cantu R, Dvorak J, et al. Summary and agreement statement of the First International Conference on Concussion in Sport, Vienna 2001: recommendations for the improvement of safety and health of athletes who may suffer concussive injuries. Br J Sports Med 2002;36(1):6-10.

31. Chen JK, Johnston KM, Collie A, McCrory P, Ptito A. A validation of the post concussion symptom scale in the assessment of complex concussion using cognitive testing and functional MRI. J Neurol Neurosurg Psychiatry 2007;78(11):1231-1238.

32. Collins MW, Field M, Lovell MR, et al. Relationship between postconcussion headache and neuropsychological test performance in high school athletes. Am J Sports Med 2003;31(2):168-173.

33. Erlanger D, Kaushik T, Cantu R, et al. Symptom-based assessment of the severity of a concussion. J Neurosurg 2003;98(3):477-484.

34. Lovell MR, Collins MW, Iverson GL, Johnston KM, Bradley JP. Grade 1 or "ding" concussions in high school athletes. Am J Sports Med 2004;32(1):47-54.

35. Piland SG, Motl RW, Guskiewicz KM, McCrea M, Ferrara MS. Structural validity of a self-report concussion-related symptom scale. Med Sci Sports Exerc 2006;38(1): $27-32$.

36. Friston KJ. Commentary and opinion. II. Statistical parametric mapping: ontology and current issues. J Cereb Blood Flow Metab 1995;15(3):361-370.

37. Fox MD, Snyder AZ, Vincent JL, Corbetta M, Van Essen DC, Raichle ME. The human brain is intrinsically organized into dynamic, anticorrelated functional networks. Proc Natl Acad Sci U S A 2005;102(27):9673-9678.

38. Salmond CH, Chatfield DA, Menon DK, Pickard JD, Sahakian BJ. Cognitive sequelae of head injury: involvement of basal forebrain and associated structures. Brain 2005;128(Pt 1):189-200.

39. Van der Werf YD, Witter MP, Uylings HB, Jolles J. Neuropsychology of infarctions in the thalamus: a review. Neuropsychologia 2000;38(5):613-627.

40. Little DM, Kraus MF, Joseph J, et al. Thalamic integrity underlies executive dysfunction in traumatic brain injury. Neurology 2010; 74(7):558-564.

41. Chu Z, Wilde EA, Hunter JV, et al. Voxelbased analysis of diffusion tensor imaging in mild traumatic brain injury in adolescents. AJNR Am J Neuroradiol 2010;31(2):340-346.

42. Kirov II, Patil V, Babb JS, Rusinek H, Herbert J, Gonen O. MR spectroscopy indicates diffuse multiple sclerosis activity during remission. J Neurol Neurosurg Psychiatry 2009; 80(12):1330-1336.

43. Ge Y, Patel MB, Chen Q, et al. Assessment of thalamic perfusion in patients with mild traumatic brain injury by true FISP arterial spin labelling MR imaging at 3T. Brain Inj 2009;23(7):666-674.

44. Stein T, Moritz C, Quigley M, Cordes D, Haughton V, Meyerand E. Functional connectivity in the thalamus and hippocampus studied with functional MR imaging. AJNR Am J Neuroradiol 2000;21(8):1397-1401.

45. Zhang D, Snyder AZ, Fox MD, Sansbury MW, Shimony JS, Raichle ME. Intrinsic functional relations between human cerebral cortex and thalamus. J Neurophysiol 2008; 100(4):1740-1748.

46. Aron AR, Schlaghecken F, Fletcher PC, et al. Inhibition of subliminally primed responses is mediated by the caudate and thalamus: evidence from functional MRI and Huntington's disease. Brain 2003;126(Pt 3):713-723.

47. Steriade M. Sleep, epilepsy and thalamic reticular inhibitory neurons. Trends Neurosci 2005;28(6):317-324.

48. Navarro X. Chapter 27: neural plasticity after nerve injury and regeneration. Int Rev Neurobiol 2009;87:483-505.

49. Garraghty PE, Kaas JH. Functional reorganization in adult monkey thalamus after peripheral nerve injury. Neuroreport 1991; 2(12):747-750.

50. Churchill JD, Arnold LL, Garraghty PE. Somatotopic reorganization in the brainstem and thalamus following peripheral nerve injury in adult primates. Brain Res 2001; 910(1-2):142-152.

51. Goldstein RZ, Tomasi D, Alia-Klein N, Zhang L, Telang F, Volkow ND. The effect of practice on a sustained attention task in cocaine abusers. Neuroimage 2007;35(1):194-206.

52. Potter DD, Bassett MR, Jory SH, Barrett K. Changes in event-related potentials in a threestimulus auditory oddball task after mild head injury. Neuropsychologia 2001;39(13): 1464-1472.

53. Ricker JH, Müller RA, Zafonte RD, Black KM, Millis SR, Chugani H. Verbal recall and recognition following traumatic brain injury: a [0-15]-water positron emission tomography study. J Clin Exp Neuropsychol 2001; 23(2):196-206.

54. Levine RA, Hung J, Otsuji Y, et al. Mechanistic insights into functional mitral regurgitation. Curr Cardiol Rep 2002;4(2):125-129.

55. Cauda F, Micon BM, Sacco K, et al. Disrupted intrinsic functional connectivity in the vegetative state. J Neurol Neurosurg Psychiatry 2009;80(4):429-431. 